\title{
Diagnosis and Management of Macular Holes Traumatic
}

\author{
Reny Violeta ${ }^{1 *}$, AK Ansyori ${ }^{1}$, Ramzi Amin ${ }^{1}$ \\ ${ }^{1}$ Department of Ophthalmology, Faculty of Medicine, Universitas Sriwijaya, Indonesia \\ *Correspondence author email : reny.violeta@gmail.com
}

\begin{abstract}
Introduction. Macular hole is a defect in the retina involving the macula and fovea, in the form of damage to full thickness from the internal limiting membrane (ILM) to the outermost part of the photoreceptor layer. It was more often found in women. In patients with myopia disorders can be found at a younger age. In this case we report the macular hole caused by blunt trauma, as was the first time a macular hole case was discovered.
\end{abstract}

Case Presentation. A man 24-year-old, Mr. C, came to Palembang RSMH eye clinic on August 15, 2014 with complaints of blurred right eye vision. Main Complaints: Right eye blurred. History Disease: Right eye blurred for about 3 months before entering the hospital. Blur is felt after the patient had a motorcycle accident 3 months ago. At that time the collision sufferer avoided the truck, the right eye hit the motorcycle handlebar, the patient then fell from the motorcycle and hit the highway head. A history of frequent headaches is denied, nausea, vomiting is denied, history of seeing in tunnels is denied. Supporting examination is Amsler Grid Oculi Dextra: there is a black hole in the middle of the image. differential diagnosis is Traumatic Macular Hole OD, Lamellar Macular Hole OD, work diagnosis is Traumatic Macular Hole stage 3 OD.

Conclusion. Traumatic macular holes can occur immediately after blunt trauma to the eyeball, the accuracy of the diagnosis and the speed of the timing for surgery, greatly determining the prognosis. Vitrectomy and combined with internal limiting membrane peeling have been shown to provide excellent results in macular surgery in macular hole cases.

Keywords. macular holes traumatic, eye trauma, diagnosis, management

\section{Introduction}

Macular hole is a defect in the retina involving the macula and fovea, in the form of damage to full thickness from the internal limiting membrane (ILM) to the outermost part of the 


\section{SRIWIJAYA JOURNALOF OPHTHALMOLOGY}

G्ञा०

photoreceptor layer. ${ }^{1-3}$ more often found in women. ${ }^{1,2,5,6}$ In patients with myopia disorders can be found at a younger age. Idiopathic age related macular holes is one of the most common causes of central visual loss. ${ }^{2}$ In 2011 it was said that 3 out of 1000 individuals suffer from idiopathic macular holes. ${ }^{2}$ The Beaver Dam Eye Study in its study observed 4926 individuals over 42 years of age, and found that full thickness macular holes occur in $0.3 \%$ of the population, and are almost entirely unilateral. But the risk of getting a macular hole in the other eye increases by $10 \%$ in the next 5 years. ${ }^{2,5,6}$

Macular holes were actually first discovered by Knapp in 1869 in patients with blunt eyeball trauma. Research at that time directed that the occurrence of macular holes was the result of blunt trauma. However, research in the modern era proves that this condition is actually caused more by atraumatic, so it is classified in two groups, idiopathic macular holes, and traumatic macular holes. Where it has been reported that $80 \%$ of macular hole cases are idiopathic, and only $10 \%$ are proven to be caused by trauma. ${ }^{1,3,14}$

Although the first official publication of the study of macular holes and their management was carried out by Henri Noyes in 1871, but more than a hundred years later, in 1991, Kelly and Wendell successfully performed the first vitrectomy to close macular holes by means of pars plana vitrectomy and membrane peeling. At present, this procedure is the standard for the management of macular holes where the success rate reaches $86 \%$ to $95 \%$ and almost all studies show a sharp increase in vision on post-operative. ${ }^{10}$

In this case we report the macular hole caused by blunt trauma, as was the first time a macular hole case was discovered. The aim is to increase our vigilance and ability as an ophthalmologist in dealing with cases of patients with blunt eyeball trauma, or with a previous history of head injury with blunt eyeball trauma, so that they can evaluate a comprehensive and appropriate ophthalmological examination in establishing a diagnosis of traumatic macular hole. In addition, we also report the operative management of vitrectomy and ILM peeling that we performed on this patient, in accordance with procedures standard macular hole management

\section{Case Report}

A man 24-year-old, Mr. C, came to Palembang RSMH eye clinic on August 15, 2014 with complaints of blurred right eye vision. Medical record number: 838349. Anamnesis (Autoanamnesis on 15 August 2014). Main Complaints: Right eye blurred. History Disease: Right eye blurred for about 3 months before entering the hospital. Blur is felt after the patient had a motorcycle accident 3 months ago. At that time the collision sufferer avoided the truck, the right 
eye hit the motorcycle handlebar, the patient then fell from the motorcycle and hit the highway head. The patient was unconscious for 1 day and was treated at the hospital, when he was aware the patient claimed his right eye immediately blurred, and swelling on the right eyelid. When the accident in the eyeball is not affected by any object, there is no blood coming out of the eyeball, there is no fluid such as egg white. Patients do not receive special treatment in the eye field during treatment. Sufferers claim to only treat the right eye with eye drops (Insto®). 1month before entering the hospital right eye complaints felt increasingly blurred, sufferers have difficulty reading closely, and difficult to return to work normally as before the accident (Work as a coal mining machine technician), then the patient went back to the hospital and was referred to RSMH.

A history of frequent headaches is denied, nausea, vomiting is denied, history of seeing in tunnels is denied. A history of seeing like a rainbow is denied, a history of seeing flying objects is denied, vision complaints such as being blinded by a curtain.

Past Disease History: History of eye glasses is absent, vision before the accident was recognized as normal - no complaints at long distance or close-range viewing. History of high blood pressure is denied, history of diabetes is denied.

Family Disease History: History of the same disease in a family is denied, a history in the family there is a glaucoma that is denied, a history of blindness in the family is denied.

Generalist status physical examination table

\begin{tabular}{|ll|ll|}
\hline General state & $:$ Good & Pulse & $: 80 \mathrm{x} /$ menit \\
Awareness & $:$ Compos Mentis & Respiratory rate & $: 18 x /$ menit \\
Blood Pressure & $: 120 / 80 \mathrm{mmHg}$ & Temperature & $: 37^{0} \mathrm{C}$ \\
\hline
\end{tabular}

Figure Ophthalmologic status

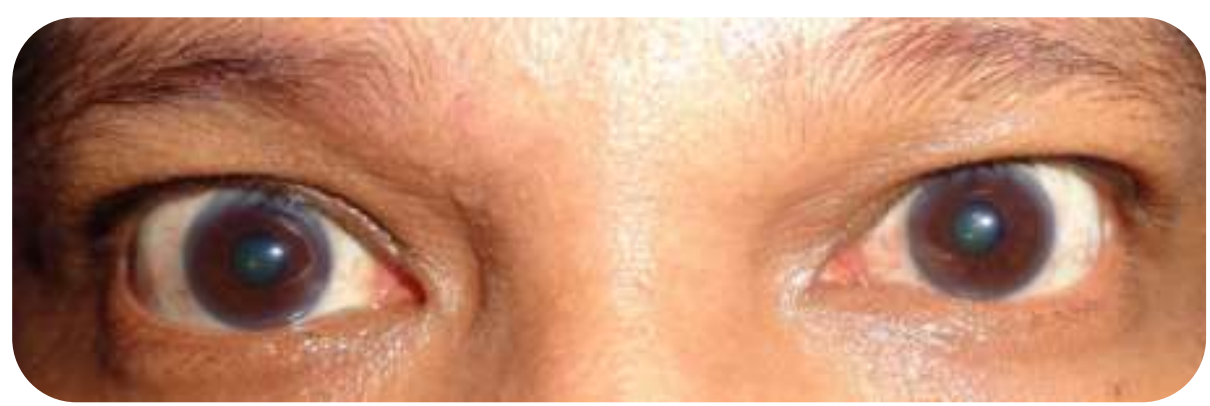

\begin{tabular}{|l|c|c|}
\hline & OD & OS \\
\hline Vision & $4 / 60 \mathrm{ph}(-)$ & $6 / 6$ \\
\hline TIO & $18,5 \mathrm{mmHg}$ & $18,5 \mathrm{mmHg}$ \\
\hline KBM & \multicolumn{2}{|c|}{ Ortophoria } \\
\hline
\end{tabular}




\begin{tabular}{|c|c|c|}
\hline GBM & & \\
\hline Palpebra & Normal & Normal \\
\hline Conjunctiva & Normal & Normal \\
\hline Cornea & Clear & Clear \\
\hline BMD & Medium & Medium \\
\hline Sliced & Good image & Good image \\
\hline Pupil & $\begin{array}{c}\mathrm{B}, \mathrm{C}, \mathrm{RC} \mathrm{L} / \mathrm{TL}(+) \mathrm{N}, \varnothing 3 \mathrm{~mm} \\
\text { RAPD (-) }\end{array}$ & $\begin{array}{c}\mathrm{B}, \mathrm{C}, \mathrm{RC} \text { L/TL (+) N, } \varnothing 3 \mathrm{~mm} \\
\text { RAPD (-) }\end{array}$ \\
\hline Lens & Clear & Clear \\
\hline $\begin{array}{l}\text { Posterior } \\
\text { Segment }\end{array}$ & RFOD $(+)$ & RFOS $(+)$ \\
\hline Papil: & $\begin{array}{l}\text { Round, firm boundary, normal red } \\
\text { color, c / d: } 0.4 \text {, a / v: } 2 / 3 \text {. }\end{array}$ & $\begin{array}{l}\text { Round, firm boundary, normal red } \\
\text { color, c / d: } 0.3 \text {, a / v: } 2 / 3 \text {. }\end{array}$ \\
\hline Macula: & $\begin{array}{l}\text { Fovea reflexes }(+) \downarrow \downarrow \\
\text { visible picture of the hole in the red } \\
\text { macula (red base) diameter } \pm 0.5 \\
\text { DD, yellow spot }(+) \text {, yellow ring } \\
(+) \text {, it appears a picture of a firm } \\
\text { gray line around the hole }\end{array}$ & Fovea reflexes $(+) \mathrm{N}$. \\
\hline Retina: & $\begin{array}{l}\text { Good blood vessel contour, } \\
\text { bleeding (-), exudate (+) around } \\
\text { the macula, PVD (+) images } \\
\text { around the macula area, visible } \\
\text { atrophy area around the hole, } \\
\text { Weiss Ring (-). }\end{array}$ & $\begin{array}{l}\text { Good blood vessel contour, bleeding } \\
(-) \text {, exudate (-) }\end{array}$ \\
\hline
\end{tabular}

Drawing retinal drawing OD

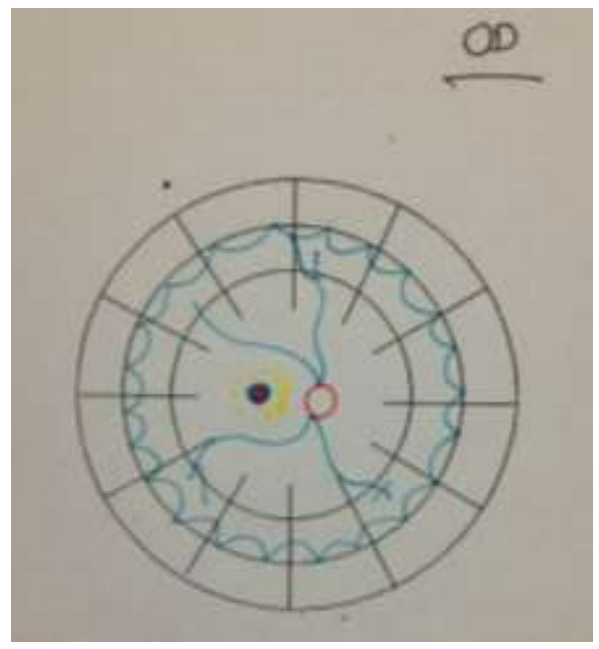




\section{SRIWIJAYA JOURNALOFOPHTHALMOLOGY}

aro

Supporting examination is Amsler Grid Oculi Dextra: there is a black hole in the middle of the image. Oculi Sinistra: within normal limits.

Fundus photo

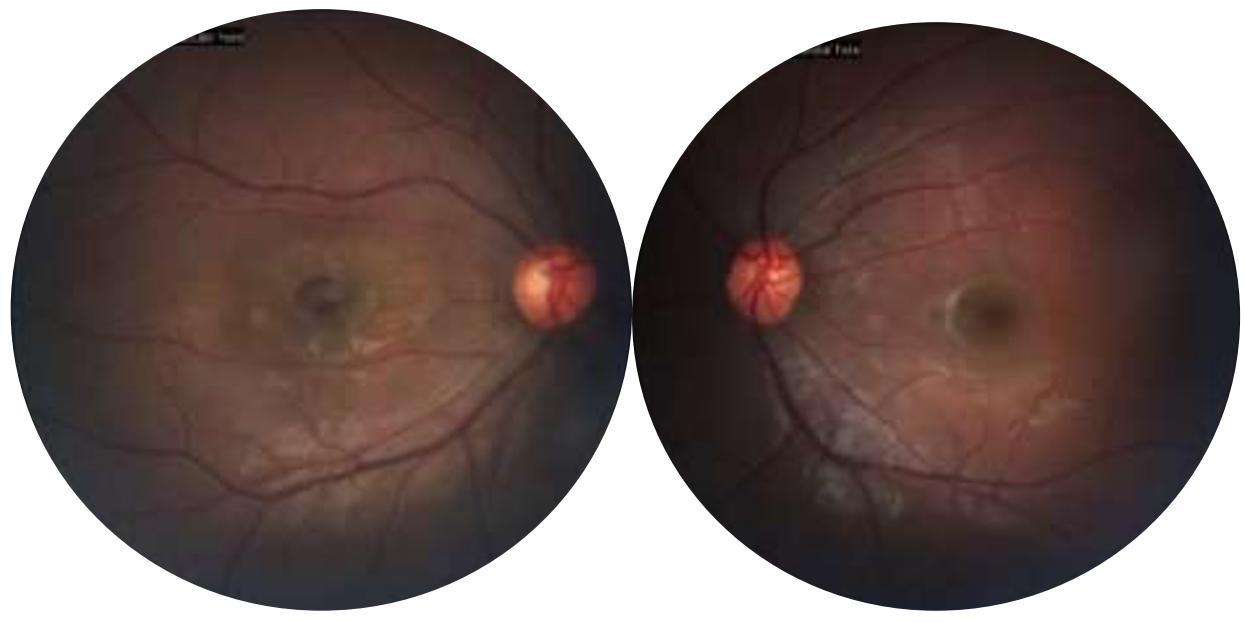

Impression: macular hole OD

Oculi dextra

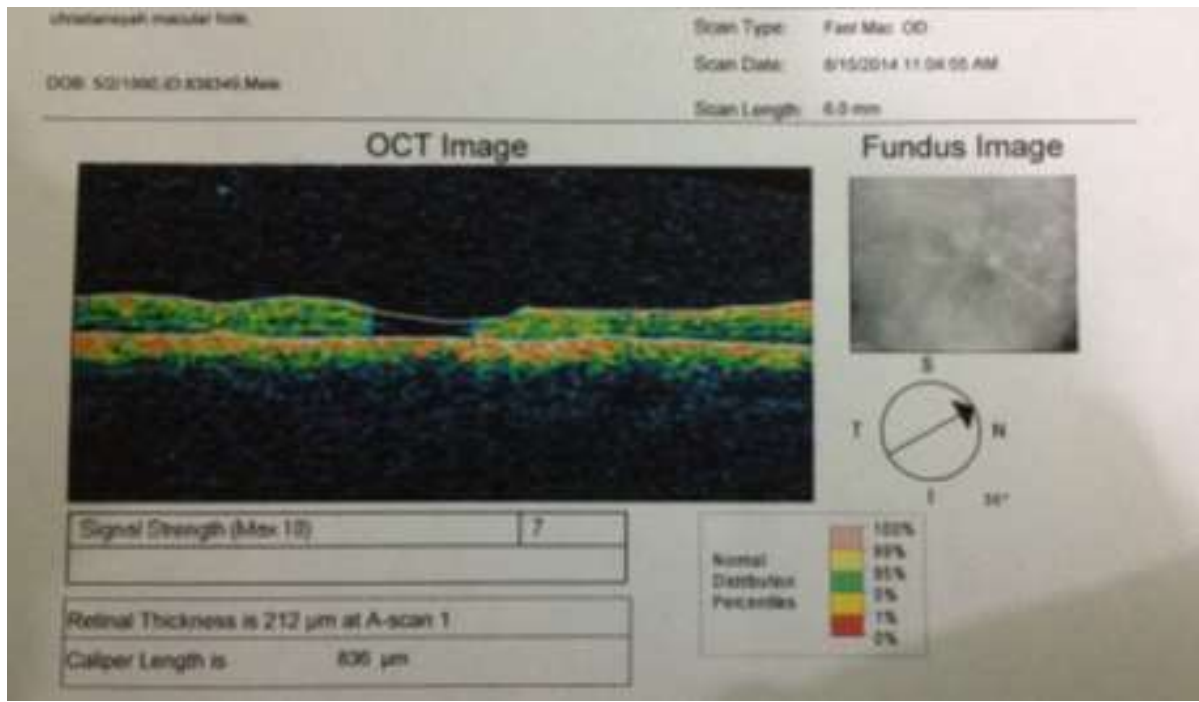

"There is a picture of hyporeflectivity in the macula, above RPE, with a diameter of 836 $\mu \mathrm{m}$, with an internal limiting membrane $(+)$, operculum $(+)$ picture"

Impression: Macular Hole stage 3

Oculi Sinistra 


\section{SRIWIJAYA JOURNALOF OPHTHALMOLOGY}

\section{बज्ञा}

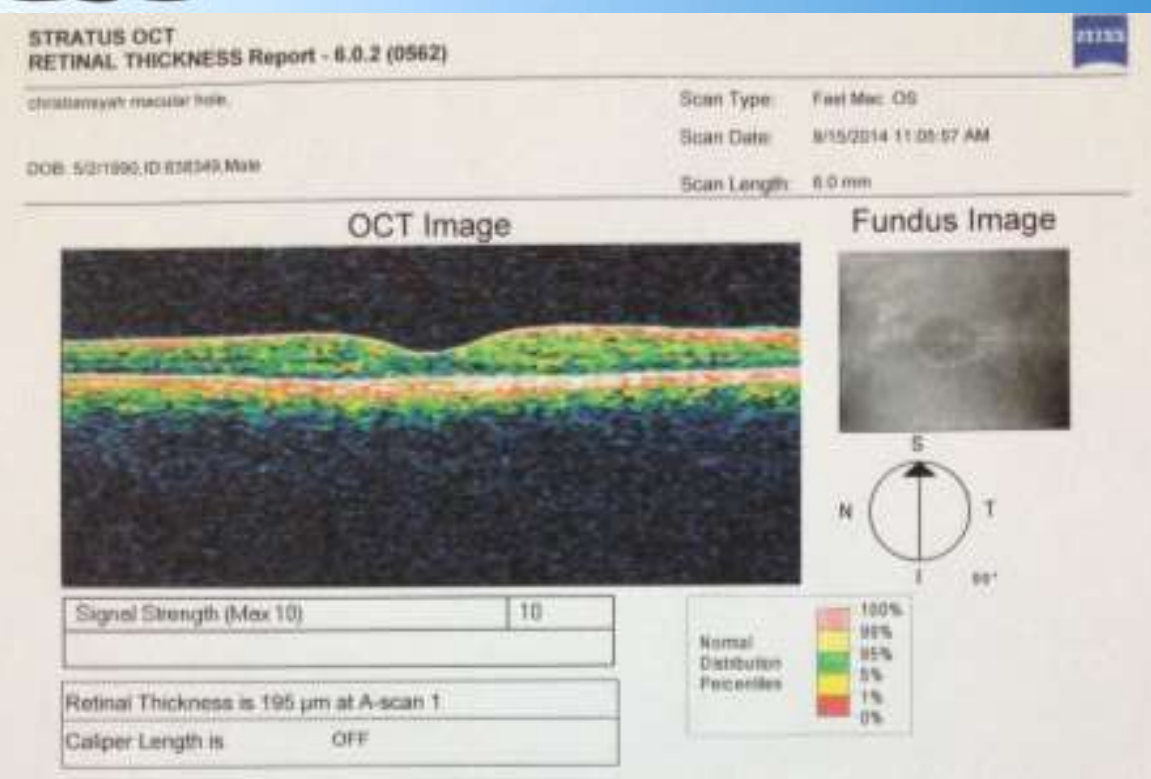

"There are areas of hyper-reflectivity among hypo-reflectivity areas, Macular Depression $(+) "$

\section{Impression: OCT Normal}

Comparative image analysis of Macular Thickness OD and OS

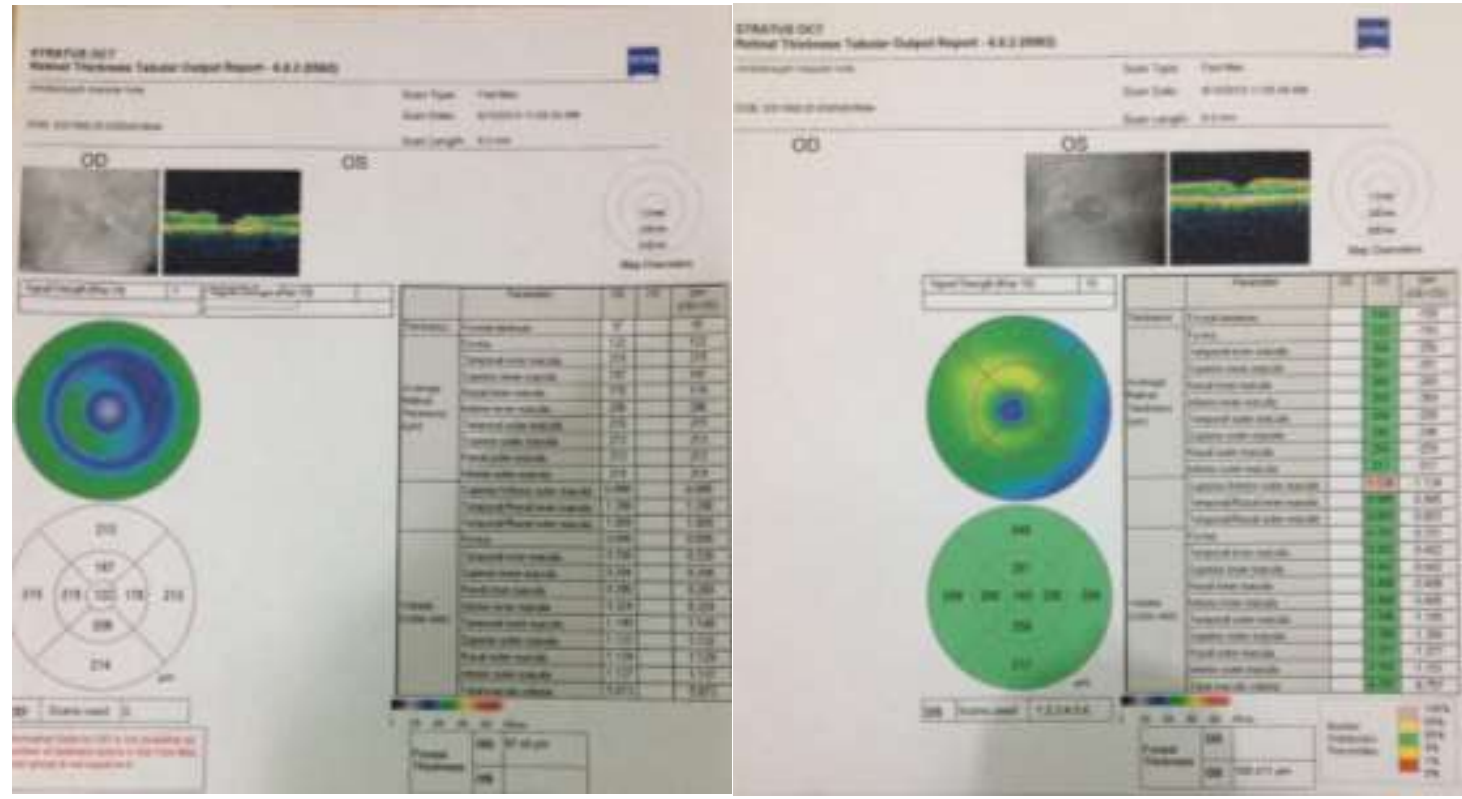

Table of laboratory results

\begin{tabular}{|l|l|l|l|l|l|}
\hline $\mathrm{Hb}$ & $:$ & $15,5 \mathrm{~g} / \mathrm{dl}$ & Platelets & $:$ & $257.000 \mathrm{~mm}^{3}$ \\
\hline Erythrocytes & $:$ & $4.570 .000 / \mathrm{mm}^{3}$ & & $:$ & \\
\hline $\mathrm{Ht}$ & $:$ & $43 \mathrm{vol} \%$ & CT & $:$ & 9 minutes \\
\hline Leukocytes & $:$ & $9.100 \mathrm{~mm}^{3}$ & BT & $:$ & 2 minutes \\
\hline
\end{tabular}




\begin{tabular}{|l|l|l|l|l|l|}
\hline & $:$ & & BSS & $:$ & $105 \mathrm{mg} / \mathrm{dl}$ \\
\hline $\mathrm{Na}$ & $:$ & $147 \mathrm{mmol} / \mathrm{l}$ & $\mathrm{K}$ & $:$ & $4.8 \mathrm{mmol} / \mathrm{dl}$ \\
\hline Ureum & $:$ & $16 \mathrm{mg} / \mathrm{dl}$ & Creatinine & $:$ & $0.94 \mathrm{mg} / \mathrm{dl}$ \\
\hline
\end{tabular}

The differential diagnosis is Traumatic Macular Hole OD, Lamellar Macular Hole OD, work diagnosis is Traumatic Macular Hole stage 3 OD. Management is informed consent, Pro Amsler Grid examination, Pro fundus photo examination, Pro OCT, Pro Vitrectomy OD and Internal Limiting Membrane Peeling OD with general anesthesia. Pro Routine blood laboratory examination and clinical chemistry, Pro X-ray piston, Pro Consultant Anesthesia, Pro Consultant Internal Medicine. Prognosis is Quo ad Vitam: dub ad ad bonam, Quo ad functionam: dubia.

\section{Discussion}

Reported a case of a 24-year-old man who came to the vitreoretinal eye clinic with a blurred right eye complaint since, 3 months ago. History of accidents - falling from a motorcycle with the head hitting a motorcycle handlebar and falling on the highway, the patient was unconscious for 1 day, and when he realized the patient claimed vision was blurred. Vision has become blurred since the past 1 months, sufferers have difficulty reading closely and work normally as before the accident. Previous history of glasses was not available, a history of blurred vision before the accident was absent.

The ophthalmology examination showed normal anterior segments in both eyes, while examination of the posterior segment of the right eye revealed a hole in the macula. When examining the function of the macula, which is the Amsler grid, the patient claims there is a black hole in the middle of the image. The examination continued with fundus and OCT photographs. OCT examination revealed a hole in the right eye macula with a diameter of $836 \mu \mathrm{m}$. Fundus and OCT images were in accordance with Gass criteria that the sufferer was included in Macular Hole Stage 3. We diagnosed patients with Traumatic Macular Hole Stage 3 OD. We plan operative vitrectomy and ILM peeling under general anesthesia.

The operative vitrectomy and ILM peeling went well, with the result of an increase in vision from preoperative $4 / 60$ to $6 / 60$ at 1 month postoperative examination. At the follow-up of the OCT examination, there was also significant progress, with the hole diameter shrinking to $598 \mu \mathrm{m}$ in the first week, $548 \mu \mathrm{m}$ in the first postoperative month, and staying $548 \mu \mathrm{m}$ in the five postoperative months. Sharp vision at 5 months postoperatively is still stable at 6/60. Although the expected 
total hole closure does not occur in this patient, a sharp increase in vision shows that with proper treatment, especially surgery, for macular holes, the eye's function as one of the human's vital senses can be saved. Not closing the hole completely in the patient is possible because the new patient comes for evaluation after 3 months post-trauma, then added to the difficulty of getting materials and surgical equipment so that the time span from the accident to the intervention of surgery becomes quite long. While the results of the study indicate that the speed of action determines the level of success and prognosis.

\section{Conclusion}

Macular holes can be grouped into two, namely idiopathic macular holes and secondary macular holes which in this case are traumatic macular holes. Macular holes consist of four stages based on Gass classification, where the staging is based on ophthalmoscopy and OCT examination.

Traumatic macular holes can occur immediately after blunt trauma to the eyeball, the accuracy of the diagnosis and the speed of the timing for surgery, greatly determining the prognosis. Vitrectomy and combined with internal limiting membrane peeling have been shown to provide excellent results in macular surgery in macular hole cases. Nearly all research proves the success of therapy with this procedure, where in postoperative OCT a hole is closed, or the hole diameter is reduced. And success can also be seen from the ability to sharply increase vision by 2 lines or more.

In the patients we report, there was progress in the postoperative vision, but the hole did not close completely. This is possible because the time span is quite long from the accident sufferer to the RSMH eye clinic for evaluation. But the results of actions taken in patients namely vitrectomy and ILM peeling, prove that this action is effective so that the sharp vision in our patients rose from $4 / 60$ to $6 / 60$ this is according to the results of existing studies that vitrectomy and ILM peeling can improve vision 2 line or more. 